\title{
Manejo de las radiaciones ionizantes en instalaciones dentales españolas: intraorales y panorámicos
}

\author{
Jodar S*, Alcaraz M**, Martínez-Beneyto Y*, Pérez L*, Velasco E***, López M*
}

\section{RESUMEN}

El objetivo de este estudio fue determinar las características de la radiología dental en la Comunidad Autónoma de Murcia, con el propósito de poder evaluar el grado de cumplimiento de las recomendaciones Oficiales de la Unión Europea. Para ello se estudian 1822 informes oficiales de control de calidad en radiodiagnóstico dental intraoral y 157 pertenecientes a exámenes panorámicos durante cinco años consecutivos.

Los resultados obtenidos ponen de manifiesto que un tercio de los equipos dentales intraorales revisados presentan averías en su funcionamiento y un 6’24\% en el caso de los panorámicos. Solamente un $63.54 \%$ de las instalaciones intraorales cumplirían con las recomendaciones oficiales al emplear equipos modernos que funcionan a $70 \mathrm{kVp}, 8 \mathrm{~mA}, 20 \mathrm{~cm}$. de distancia foco piel y $1.5 \mathrm{~mm}$ de Al. Se ha producido un descenso del $11.53 \%$ en las dosis medias administradas a lo largo del estudio, aumentando la empleada con aparatos panorámicos.

La entrada en vigor de esta nueva legislación ha supuesto un descenso en las dosis medias de radiación administradas a los pacientes tras los cinco años de evolución analizados aunque se sigue incumpliendo las recomendaciones oficiales europeas sobre Protección Radiológica.

Palabras clave: Radiodiagnóstico, radiografía dental, dosis medias de radiación, control de calidad.

\section{ABSTRACT}

The aim of the study was to determinate Dental Radiology behaviour in Murcia Community to control the European Union Recommendations. 1822 official reports on quality assurance in intraoral radiology and 155 official reports on panoramic radiology have been studied.

The results indicate that one and a half of intraoral radiographic equipments studied and 6`2 $24 \%$ in panoramic had alterations. Only the $63^{\prime} 54 \%$ of the surgeries achived the European Community Recommendations of intraoral radiology working to $70 \mathrm{kVp}, 8 \mathrm{~mA}$ and $1.5 \mathrm{~mm}$ of $\mathrm{Al}$. There have been a decrease of the intraoral radiation dose exposured.

The recent legislation have meant a decrease in the intraoral radiation dose administrated despite of the unachivement of European Community Recommendations.

Key words: Radiodiagnosis, dental radiography, mean doses, assurance quality.

Aceptado para publicación: abril 2004.

* Area de Estomatología. Facultad de Medicina/Odontología. Universidad de Murcia.

** Area de Radiología y Meedicina Física. Facultad de Medicina/Odontología. Universidad de Murcia.

*** Unidad Técnica de Protección Radiológica Asigma S.A.L.

Jodar S, Alcaraz M, Martínez-Beneyto Y, Pérez L, Velasco E, López M. Manejo de las radiaciones ionizantes en instalaciones dentales españolas: intraorales y panorámicos. Av. Odontoestomatol 2005; 21-1: 361-370. 


\section{INTRODUCCIÓN}

La organización de Naciones Unidas en el último informe publicado por su Comité Científico sobre los efectos de la radiación atómica describe que la radiografía dental es una de las exploraciones radiológicas más frecuentes (1).

La estimación global anual para el radiodiagnóstico dental, es de unos 520 millones de exploraciones radiológicas dentales en todo el mundo, que se corresponde con una frecuencia de 90 exploraciones/1000 habitantes. Sin embargo, se asume una distribución que pone de manifiesto que el $90 \%$ de las exploraciones dentales se realizan en los países de nivel I o bien desarrollados, entre los que se encuentra España.

Se ha estimado que el Radiodiagnóstico Dental contribuye con una extensa parte a la dosis colectiva anual global con una dosis sobre la población mundial de aproximadamente $14.000 \mathrm{~Sv}$ /hombre, que equivale a $0.002 \mathrm{mSv} /$ persona. Aproximadamente, el $68 \%$ de esta dosis colectiva global se englobaría dentro de los países de nivel I.

En dicho informe, se puso de manifiesto que los equipos de radiodiagnóstico dental se estiman en 440 equipos/millón de personal, lo que equivale aproximadamente al $57 \%$ de todos los equipos de radiodiagnóstico médico. Además, el numero anual global de exploraciones radiológicas dentales se estima en 310.000 exploraciones/millón de habitantes, que equivaldría al $25,20 \%$ de todas las exploraciones radiológicas con rayos $\mathrm{X}$ de las realizadas en la población mundial. En definitiva casi la mitad de los generadores de los equipos de radiodiagnóstico son dentales, aún cuando solo una cuarta parte de todas las exploraciones radiológicas corresponderían al campo odontológico.

Por otra parte destacar, que el número de profesionales expuestos a radiación ionizante con control dosimétrico dentro del campo odontológico ha ido aumentando a lo largo del tiempo, desde 10.026 trabajadores en 1998 hasta 12.064 en el año 2002 (2) y estos suponen el $22 \%$ de todos los profesionales que trabajan en el radiodiagnóstico.

Si se consideran únicamente los Trabajadores Profesionalmente Expuestos con dosis significativas, y se excluyen los casos de potencial sobre-exposición, la dosis individual media en Odontología resulta ser el doble que la producida por el radiodiagnóstico general: $1.12 \mathrm{mSv} / \mathrm{año}$ en Odontología frente a 0,69 mSv/año en radiodiagnóstico general.

Todo ello constituye una situación radicalmente opuesta a la percepción del riesgo en OdontoEstomatología percibido por los propios profesionales respecto de la situación actual, ya que las personal controladas que más se irradian en el empleo de rayos $\mathrm{X}$ con fines médicos lo constituyen los trabajadores que desarrollan su actividad profesional en Odontología.

En síntesis, podría decirse que la actividad del odonto-estomatólogo español durante décadas ha sido la de asumir un riesgo frente a las lesiones inducidas por radiaciones ionizantes de la utilización de sus equipos de rayos $\mathrm{X}$ prácticamente nulo, sobre todo en base a su suposición de recibir escasísimas dosis de rayos $\mathrm{X}$ como consecuencia de su trabajo. Actitud, que se traslada con facilidad al personal que trabaja bajo sus ordenes en las clínicas dentales, y como consecuencia se refleja en el trato y actitudes de protección radiológica con sus pacientes.

Sin embargo, los resultados que se van obteniendo durante los últimos años ponen de manifiesto que los profesionales del radiodiagnóstico dental son los que más se irradian de todo el radiodiagnóstico médico contribuyendo de una forma muy significativa a la dosis colectiva de la población y constituyendo de forma sorprendente y significativa los casos de trabajadores que pueden superar las dosis máximas permitidas para los Trabajadores Profesionalmente Expuestos.

En el año 2002 se asume una población de 39,8 millones de españoles, con 109.520 médicos especialistas de los que 7.064 son médicos especialistas encargados de los diferentes procedimientos radiológicos médicos. Junto con ellos se establece en 12.414 el número de Odontólogos y Estomatólogos en nuestro país, que supone 1 odontólogo/ 3.206 habitantes.

El numero de equipos de radiodiagnóstico general se sitúa en 14.411 equipos de los que 7.327 son 
equipos de rayos $\mathrm{X}$ dentales por los que suponen el $50,8 \%$ de todos los equipos de radiodiagnóstico.

Sin embargo, el número debe ser significativamente mayor porque:

- El 95\% de las instalaciones radiológicas dispone de un equipo de radiología intraoral.

- Un significativo numero de instalaciones tiene más de un equipo de radiología dental.

- Numerosos odontólogos son a nivel privado, responsables de más de una instalación que dispone instalación radiológica.

En nuestro país, el numero de exploraciones o exámenes anuales de radiodiagnóstico se ha situado en 25.058.622, lo que supone una tasa de 629 exámenes /1000 habitantes $(3,4)$.

Junto con ello, el número de exploraciones de radiodiagnóstico dental que se realizan en España se ha establecido en 5.226.823/30.285.445. Considerando este valor como correcto, el número de exámenes dentales sería de 131 exploraciones /1.000 habitantes. Sin embargo, tampoco parece una cifra que pueda coincidir con la realidad de la actuación profesional odontológica ya que significaría que cada odontólogo que se dedica profesionalmente a su ocupación y considerando como profesionalmente expuesto, con todas las responsabilidades que ello implica realizar 1.1 radiografía/día.

A pesar de todo, se ha aceptado internacionalmente que los bajos niveles de dosis que individualmente se administran a los pacientes dentales se ven influenciadas significativamente por los equipos y las técnicas empleadas y por las medidas de control de calidad utilizadas en cada instalación (1).

Nuestro estudio va a permitir el análisis de forma individualizada de la actuación radiológica de una total de 1.822 informes oficiales de instalaciones radiológicas intraorales y 157 informes oficiales de instalaciones de radiología panorámica durante 5 años consecutivos, instalaciones ubicadas en la Comunidades Autónoma de la Región de Murcia con el objetivo de poder determinar el grado de cumpli- miento de las Recomendaciones Oficiales Europeas sobre Protección Radiológica.

\section{MATERIAL Y MÉTODO}

En Diciembre de 1995 se publicó un Real Decreto (2071/1995) (5), previa transposición de las directrices europeas $(6,7,8)$ en el que con carácter de Norma Básica Sanitaria obligaba a realizar un control de calidad anual a cada una de las instalaciones radiológicas españolas que se encontraban en funcionamiento. Desde entonces han sido numerosas las clínicas dentales que se han ido sumando por ley a dicha inspección, realizada por Unidades Técnicas de Protección Radiológica, empresas privadas homologas y auditadas por el Consejo de Seguridad Nuclear.

En nuestro estudio, se han analizado 1822 informes oficiales de control de calidad pertenecientes a instalaciones intraorales y 157 a panorámicos ubicados en la Comunidad Autónoma de la Región de Murcia. Dichos informes han sido proporcionados por la U.T.P.R Asigma S.A.L., y han sido elaborados desde 1996-97 hasta el año 2001, permitiendo de esta manera poder analizar la evolución llevada a cabo por las instalaciones dentales a lo largo de estos cinco años de estudio.

De dichos informes se han recogido las variables relacionadas tanto con la instalación radiológica como con todo el proceso de revelado de la película radiográfica, y así como la dosis media de radiación empleada sin tener en cuenta el valor de retrodispersión. Para la medición de dicho valor se ha realizado con tiempos correspondientes a la exploración del segundo molar superior en el caso de la intraoral, y en la radiología panorámica la dosis de referencia se ha determinado para la telerradiografía, medida a 2 metros de distancia.

Posteriormente se ha realizado un estudio estadístico para determinar el grado de dependencia y correlación de algunas variables entre sí. Para ello se ha realizado una comparación de grupos mediante un Análisis de Varianza complementado con contrastes de igualdad de medias con el método de la mínima diferencia significativa $(p<0.05)$. 


\section{RESULTADOS}

Han sido numerosas las localidades pertenecientes a la Comunidad Autónoma de la Región de Murcia recogidas en el estudio. Destacar que en 1996-97, Murcia capital suponía el $34.53 \%$ de los informes revisados, valor que ha disminuido hasta un $32.28 \%$ cinco años posteriores.

A lo largo de los cinco años de estudio se ha observado un aumento en el número de instalaciones radiológicas intraorales que emplea equipos de la marca Trophy, situándose cerca del $60.34 \%$ para el año 2001 (ver Tabla I).En el caso de los panorámicos, las marcas encontradas han sido más diversas, señalando que para el 2001 , cerca del $25 \%$ de las instalaciones pertenecían a la casa Trophy, situación compartida por Planmeca.

Con respecto a los parámetros físicos destacar que un $63.54 \%$ de los equipos dentales intraorales revisados durante el año 2001 funcionaría a valores de $70 \mathrm{kVp}$, valor muy superior a los $45.61 \%$ del primer año de estudio, lo cual implica una mejoría del 17.93 \% durante este periodo, mejoría en cuanto al cumplimiento de las recomendaciones oficiales de

\section{TABLA 1.- DISTRIBUCIÓN DE LAS MARCAS DE LOS EQUIPOS DE RADIODIAGNÓSTICO EN EL AÑO 2001}

\begin{tabular}{|c|c|c|}
\hline Marca & $N^{\circ}$ de aparatos & $\%$ \\
\hline TROPHY & 245 & 60,34 \\
TAKARA-BELMONT & 43 & 10,59 \\
GENDEX- & 34 & 8,37 \\
SATELEC & 26 & 6,4 \\
PHILIPS & 20 & 4,92 \\
ARDET & 17 & 4,18 \\
CASTELLINI & 6 & 1,47 \\
VILLA & 5 & 1,23 \\
FIAD & 3 & 0,73 \\
OTROS & 3 & 0,73 \\
CIAS & 2 & 0,49 \\
SIN IDENTIFICAR & 1 & 0,24 \\
SIEMENS & 1 & 0,24 \\
\hline TOTAL & 406 & $\mathbf{1 0 0 , 0 0}$ \\
\hline
\end{tabular}

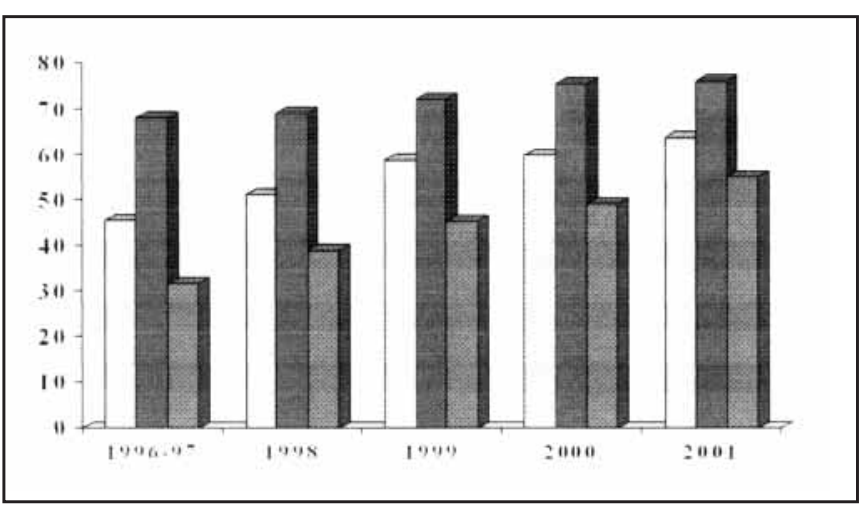

GRÁFICA 1. Evolución en el cumplimiento de las recomendaciones oficiales (70 kVp, $8 \mathrm{~mA}$ y $2,5 \mathrm{~mm} \mathrm{Al}$ ).

emplear equipos dentales radiológicos que funcionen $70 \mathrm{kVp}$ (ver Gráfica 1). Situación que también parece reflejarse con los panorámicos, ya que durante los cinco años que ha durado el estudio, se ha observado un aumento en el $18.59 \%$ de las instalaciones que funciona a $80 \mathrm{kVp}$.

Se ha encontrado una gran variabilidad en las longitudes de cono descritas en los aparatos de radiologia intraoral (ver Gráfica 2). Tal dispersión ha ido disminuyendo a lo largo de los cinco años de estudio limitándose a un $89.65 \%$ las que cumplen las recomendaciones europeas para el año 2001 (ver Gráfica 3).

Se han detectado averías en un tercio de los equipos intraorales revisados para el año 2001, averías en alcanzar el kilovoltaje, tiempo de exposición, rendimiento y linealidad marcado por el fabricante (ver Gráfica 4). En el caso de los panorámicos, destacar

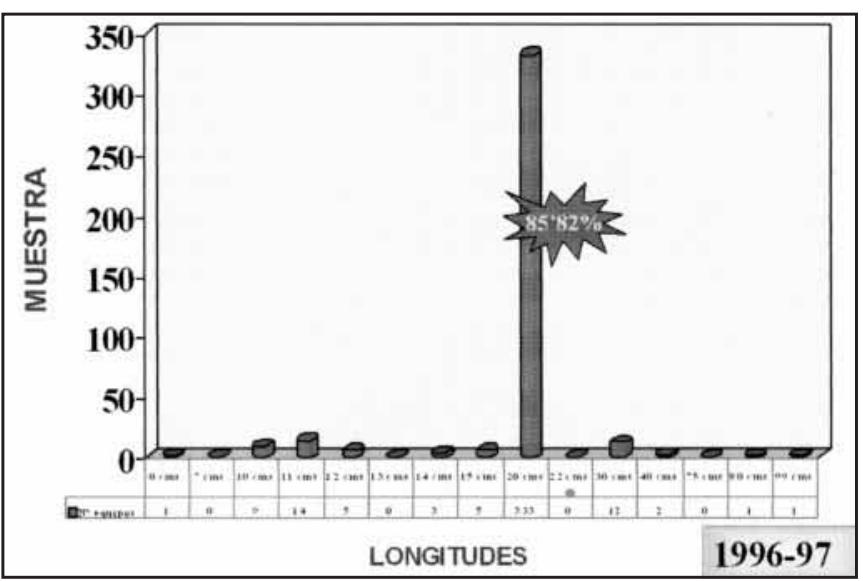

GRÁFICA 2. Exposición de las distintas longitudes de colimador empleadas en los equipos revisados durante el año 1996-97. 


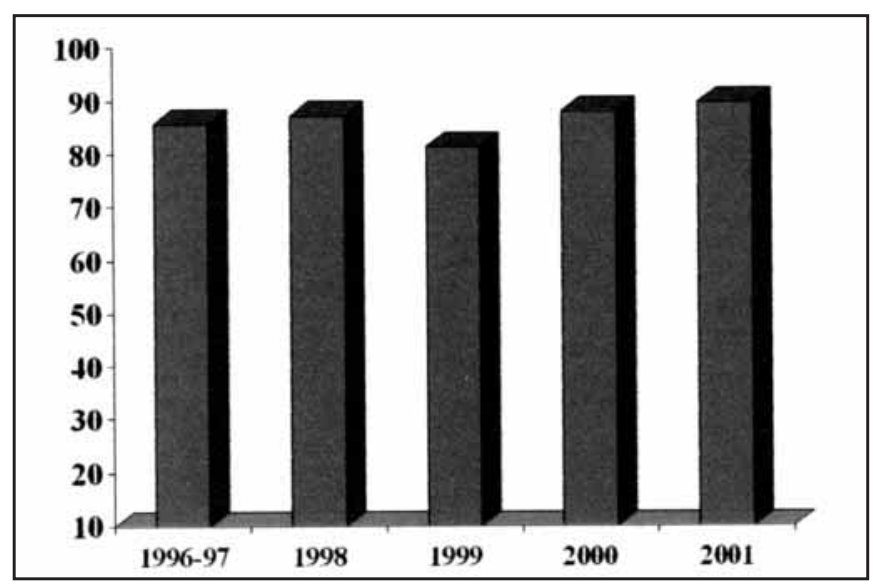

GRÁFICA 3. Evolución de la utilización de colimadores de 20 cm de longitud.

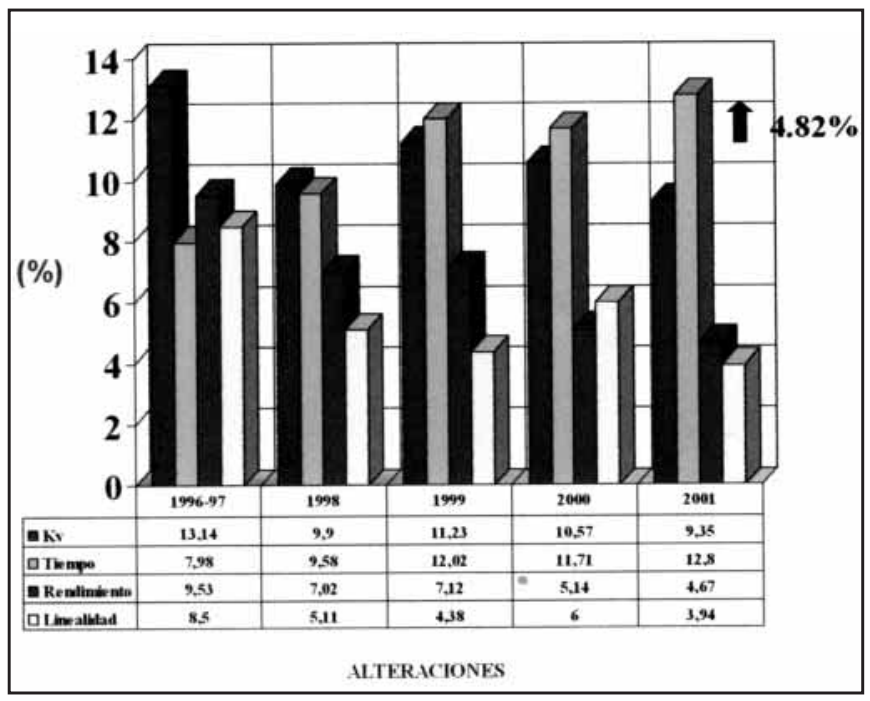

GRÁFICA 4. Alteraciones en el funcionamiento de los equipos.

que para el año 2001 , eran un $6.24 \%$ los equipos que presentaban fallos en el kilovoltaje y rendimiento del aparato.

Solamente un $9.53 \%$ de las clínicas dentales disponen de pulsadores situados fuera de la sala donde se realiza el disparo, y un $90.2 \%$ emplea cables alargadores o extensibles de 2 metros de longitud, el resto de las instalaciones utiliza situaciones totalmente ilegales (ver Gráfica 5). En el año 2001, las instalaciones con pulsadores fuera de la sala han aumentado hasta un total del $24.13 \%$.

El revelado radiográfico empleado es prácticamente manual en un $58 \%$ durante la primera revisión situación contraria al proceso de revelado efectuado en la

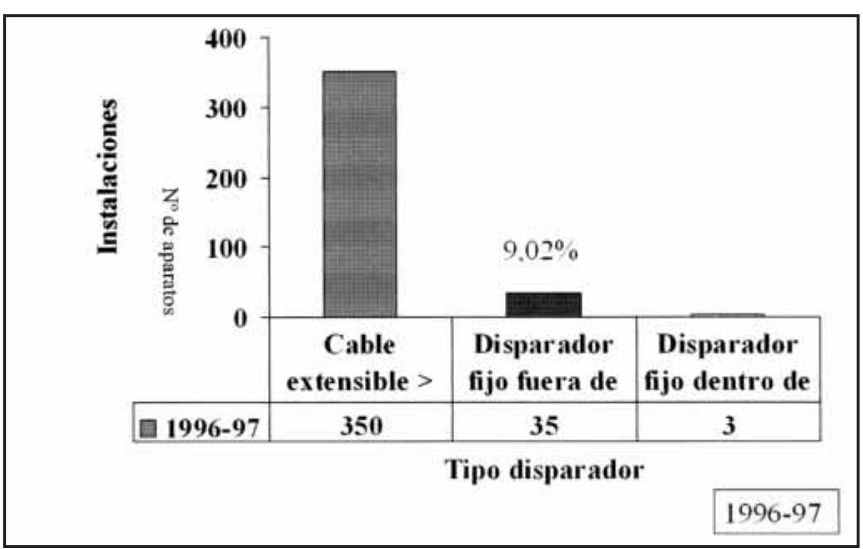

GRÁFICA 5. Forma de realizar la exposición.

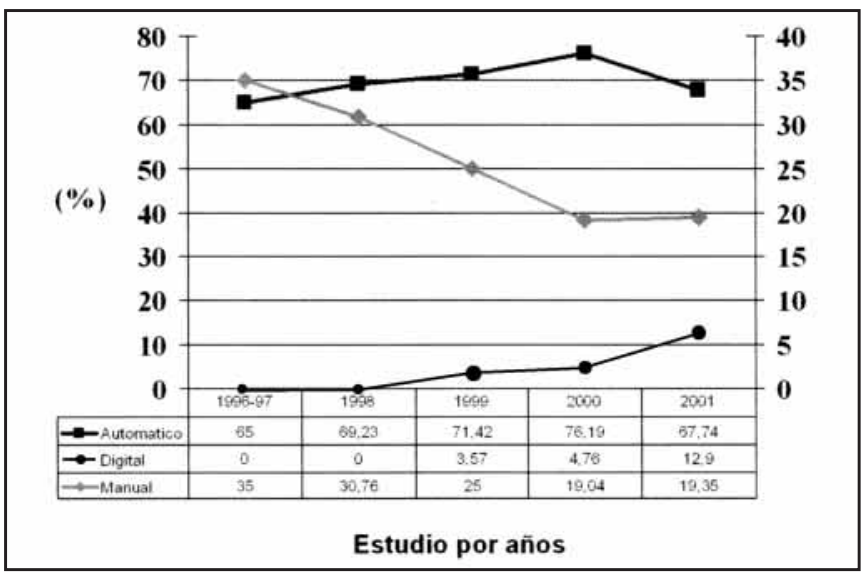

GRÁFICA 6. Revelado radiográfico.

radiografía panorámica donde el $65 \%$ de las instalaciones lo hacen automáticamente (ver Gráfica 6). Prácticamente esta situación apenas a variado durante los años de estudio, salvo la incorporación de sistemas digitales de imagen que para el año 2001, eran un $12 \%$ aproximadamente las instalaciones tanto intraorales como panorámicas que disponían de este sistema (ver Gráfica 7).

Sin embargo, en el año 2001, solamente se renuevan los líquidos de revelado semanalmente en un $80.05 \%$ de las instalaciones intraorales, y mensualmente un $44.44 \%$ según indicaciones de fabricante y se realiza un control de los líquidos de revelado, lavado y fijado en el $23.75 \%$ de las instalaciones.

La película radiográfica dental más empleada tanto para intraoral como panorámica es la KODAK, donde un $74.13 \%$ de las clínicas dentales revisadas en el año 2001, disponían de Ultraspeed (D-speed), 


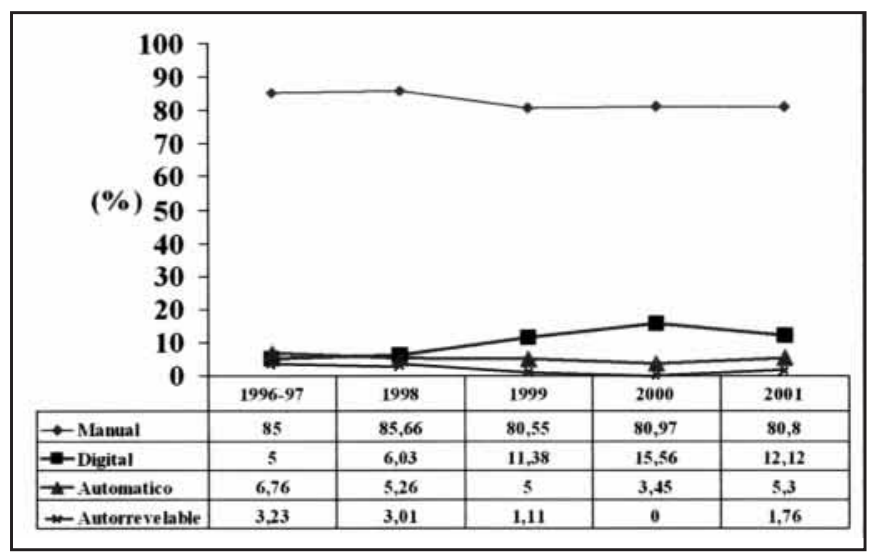

GRÁFICA 7. Revelado radiográfico intraoral.

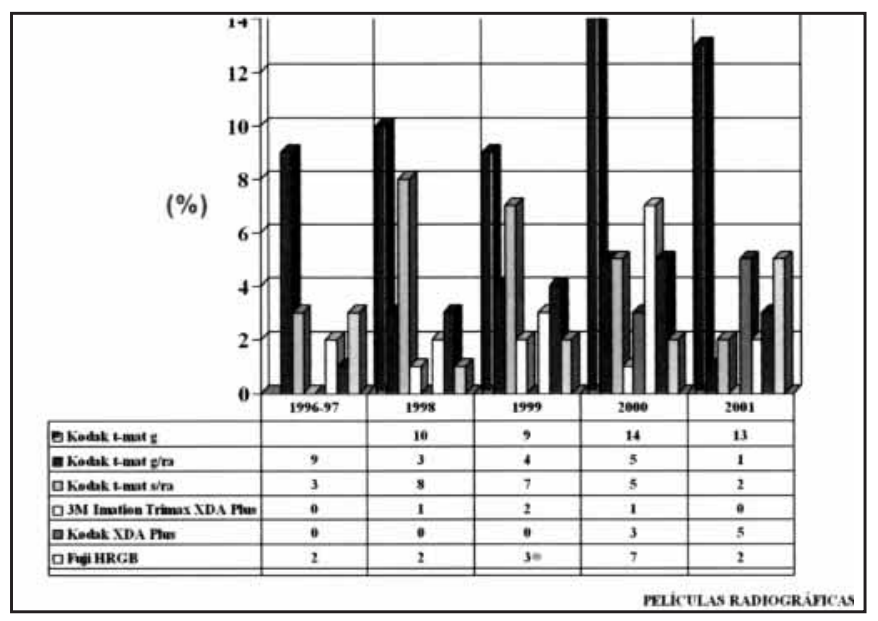

GRÁFICA 8. Películas radiográficas panorámicas.

y de la película T-mat $g$ en el caso de las panorámicas (ver Gráfica 8).

La dosis media de radiación empleada por las instalaciones de radiodiagnóstico intraoral para el año 1996-1997 es de 3.38 mGy y un $93.04 \%$ de las instalaciones cumpliría con las recomendaciones oficiales de utilizar dosis inferiores a los 7 mGy. Esta situación ha mejorado a lo largo del estudio, estableciéndose en 2.99 mGy, la dosis media para el año 2001. Estos resultados muestran un descenso del $11.53 \%$ en las dosis medias administradas. Esta situación no se refleja para los panorámicos, donde se ha producido un incremento considerable en las dosis medias de referencia para la realización de una telerradiografía y a una distancia de 2 metros, en donde la dosis ha pasado de 0.17 mGy en el año de inicio hasta un total de 0.24 mGy para el año 2001.
Los resultados obtenidos del análisis estadístico correspondiente a los informes intraorales muestran que en el mercado existen dos marcas comerciales (Castellini y villa) que ante las mismas condiciones están emitiendo dosis de radiación significativamente $(p<0.05)$ superiores al resto de marcas. Se han detectados dosis de radicación significativamente inferiores $(p<0.05)$ entre los equipos de radiología dental que cumplen con las recomendaciones oficiales de funcionar a $70 \mathrm{kVp}$ y el resto de aparatos.

El empleo de líquidos de revelado manual con una renovación semanal disminuye las dosis de radiación significativamente $(\mathrm{p}<0.001)$ con respecto a las instalaciones que lo hacen con un tiempo más prolongado.

Se han detectado diferencias significativas $(p<0.001)$ en las dosis de radiación empleadas con radiovisiografía digital y con el resto de procesos de revelado, lo que implica que el sistema digital de imagen reduce significativamente las dosis de radiación administradas comparadas con otros sistemas convencionales de procesado radiográfico (manual, automático, y resto de películas radiográficas convencionales.).

\section{DISCUSIÓN}

Parece evidente el número excesivo de exploraciones dentales que se estás realizando diariamente. En España, se estima un total de 131 exámenes/1000 habitantes, esta es inferior a la de la mayoría de los países de la Unión Europea $(3,4)$. En el Reino Unido, el número de instalaciones dentales se sitúa en 39 consultas/100.000 habitantes, con un total de 18 millones de exploraciones radiográficas anuales (9).

Además del número excesivo de radiografías dentales, existes datos que muestran que una parte importante de estas no son válidas para un buen diagnóstico clínico, situación peligrosa que hace que aumente considerablemente la dosis administrada al paciente $(10,11,12,13,14,15,16)$. Esta situación puede ser atribuible a que dichas exploraciones radiológicas han sido efectuadas con equipos anti- 
cuados, con averías, técnicas obsoletas y alteraciones o manejo incorrecto en la técnica de revelado radiográfico.

Bajo estas circunstancias hemos creído conveniente evaluar situación en la que se encuentran nuestros equipos radiológicos y las técnicas de revelado desarrolladas.

En este sentido destacar que los aparatos de radiología dental intraoral y panorámica descritos en nuestro estudio son muy similares a los del resto de países europeos, destacando incluso una cierta mejoría en el cumplimiento de las recomendaciones oficiales con respecto a otros países, en donde existe un elevado número de equipos radiológicos dentales intraorales que funcionan a valores extremos de 45 y 90 kVp $(17,18)$, situación que se repite en países como Siria, donde un $45 \%$ de los equipos funcionan con kilovoltajes de 50. Esta situación dista mucho de la descrita en Estados Unidos, donde prácticamente el $88 \%$ de los aparatos dentales funcionan a $70 \mathrm{kVp}(19)$.

En nuestro medio, cerca de un tercio de los equipos radiológicos dentales intraorales funcionan incorrectamente, situación algo inferior a la producida con los panorámicos (6.24\%).

Aunque esta situación ha mejorado con la instauración de la legislación vigente sobre control de calidad, todavía es demasiado elevado el número de instalaciones con averías en el año 2001. Esto puede ser debido al escaso servicio de mantenimiento aportado por las propias marcas comerciales que posibilita la duración de dichas averías. Pero es el propio titular de la instalación dental quién debe asumir la responsabilidad plena de eliminar esta serie de alteraciones que producen aumento en las dosis administradas a los pacientes (20).

En nuestro entorno, solamente un $63.54 \%$ de las instalaciones revisadas durante el año 2001 dispone de equipos modernos que cumplirían con las recomendaciones oficiales de la Unión Europea en cuanto a $70 \mathrm{kVp}, 20 \mathrm{~cm}$. de distancia foco-piel, 8 miliamperios y $1.5 \mathrm{~mm}$ de Al de filtración, valor ligeramente inferior al descrito en el resto del país (72.79\%) (21).
En España, para obtener la certificación y superar el control de calidad, todas las instalaciones radiológicas dentales deben de poseer algún tipo de material de protección personal. La recomendación es la de poseer dos prendas de protección diferentes, ya que en un mismo momento, profesional y paciente pueden necesitarlo al mismo tiempo. En muy pocas ocasiones se ha incorporado este dato en las publicaciones revisadas.

Recientemente, Geist Y Katz (19) han descrito que en una encuesta realizada a 65 escuelas y facultades de Odontología ubicadas en Estados Unidos y Canadá, el $95 \%$ de las mismas dispone de prendas y vestimentas de protección personal para la radiología extraoral (panorámica) y un 85 \% disponen de collares tiroideos para la radiología intraoral. Estos mismos autores sostienen que en radiología intraoral no resulta eficaz la utilización de los delantales plomados y que el "collarín tiroideo" es la prenda de protección personal del paciente más interesante para la disminución de la dosis de radiación en radiología intraoral.

Sin embargo, el "collarín tiroideo" no conlleva la eliminación de la dosis de radiación administrada a la glándula tiroides o a las glándulas submaxilares, ya que su irradiación se produce como consecuencia de quedar incluida dentro del haz primario de radiación, y no por la radiación dispersa producida. Por ello, quizás la mejor protección específica sería el denominado "escudo submandibular", y que parece especialmente diseñado para radiología intraoral pero que no se encuentra comercialmente disponible en España.

Aunque algunos delantales plomados utilizados en las clínicas odontológicas que hemos analizado, llevan una pequeña zona para actuar como "collarín", resultan completamente insuficientes. Esta situación es radicalmente diferente a lo descrito en Estados Unidos y Canadá en donde el 85 \% de las instalaciones lo utilizan habitualmente (19), aún a costa de eliminar los delantales de protección personal. En ninguno de los estudios revisados en la bibliografía se ha descrito la utilización del escudo submandibular. Este "escudo submandibular" para protección radiológica no se encuentra disponible comercialmente en nuestro país. 
El procesado automático de la película radiográfica no es el método más utilizado en nuestro entorno (5.3\%), ya que aproximadamente un $80.8 \%$ del revelado de la película radiográfica en radiología intraoral es manual. Esta situación podría hacer que aumentara significativamente el número de errores en el manejo de la técnica (control en la renovación de los líquidos de revelado, control en los tiempos de revelado, lavado y fijado, temperatura de los líquidos, etc.). Los resultados obtenidos son similares a los descritos en el resto del país (81.27 \%) (21), así como a los descritos en Grecia (22). Aunque existen países donde predomina el empleo de procesadoras automáticas como por ejemplo Dinamarca donde el $50 \%$ del revelado es automático (23), Suecia (88\%) (24) y Canadá (93 \%) (18).

Todavía en el año 2001, se ha encontrado que la película radiográfica más utilizada es la Ultraspeed (74.13\%), a pesar de ser la película más antigua en uso de la casa Kodak, la menos sensible y la que más dosis de radiación requiere. Sin embargo, la película Ektaspeed, solamente la emplean un $20.68 \%$ de las instalaciones.

En la actualidad, la casa Kodak ha retirado del mercado la película de sensibilidad E (Ektaspeed), con el objetivo de incrementar las ventas de su más reciente tipo de película (Insight). El problema que se está encontrando es que económicamente es muy superior a Ultraspeed, la película con más ventas de la casa Kodak, lo que implica que el odontólogo se niega a cambiar de película radiográfica. Además los aparatos modernos de radiología intraoral, no están preparados para intervalos tan cortos de tiempo requeridos por la película Insight, lo que implica que en numerosas ocasiones la imagen obtenida con la película Insight aparezca ennegrecida debido a un exceso de dosis de exposición.

Esta situación difiere de la descrita en otros países como Dinamarca (23), Canadá (25), Grecia (22) y Estados Unidos, donde incluso llega a emplearse mayoritariamente en un $86 \%$ de las clínicas dentales la película Ektaspeed (19)

El revelado radiográfico en la radiología panorámica muestra una situación contraria a la radiología intraoral ya que la revisión del año 2001 pone de manifiesto que en el $67.74 \%$ de las instalaciones revisadas en nuestra comunidad utiliza un revelado automático, porcentaje ligeramente superior al $65 \%$ mostrado en la primera revisión. Las películas de la casa Kodak, en sus distintas versiones (kodak Tmat $\mathrm{g} / \mathrm{ra}$ y s/ra) son las más utilizadas en $67.72 \%$ de las instalaciones revisadas en el año 2001, no sufriendo prácticamente variaciones en su empleo; el $90.91 \%$ de las pantallas de refuerzo utilizadas para la realización de la ortopantomografia corresponde a la casa Kodak en sus distintas modalidades (Kodak Lanex, Kodak Lanex Médium y Kodak Lanex Regular) durante la primera revisión efectuada, llegando en la revisión del año 2001 al 92.6\% del total de las instalaciones; de este modo la utilización tanto de las películas como de las pantallas de refuerzo de la casa Kodak no sufre prácticamente variaciones a lo largo de los cinco años de revisión en la Comunidad Autónoma de la Región de Murcia.

La dosis media de radiación descrita en nuestro estudio para el radiodiagnóstico intraoral (2.9 mGy)es ligeramente inferior a la descrita para el resto del país $(26,21,27)$ y de otros países miembros de la Unión Europea donde los valores oscilan desde los 3.5 mGy hasta 6.9 mGy $(28,22,28)$. Para la radiología panorámica, la dosis de referencia se establece en 65 mGy para el Reino Unido (30).

Toda esta situación hace que nos planteemos si las medidas adoptadas por los profesionales de la radiología dental española son suficientes para una buena protección frente a radiaciones ionizantes. Ya no hacia el paciente, sino hacia el propio profesional que como ya se ha observado maneja dosis individuales relativamente elevadas.

Aunque se han producido mejorías a lo largo de estos años, como la reducción en las dosis medias administradas, las recomendaciones oficiales quedan lejos de cumplirse en su totalidad, existiendo un gran número de instalaciones averiadas, instalaciones que han sido dadas de alta ante el Consejo de Seguridad Nuclear. A pesar de esta situación, se está desarrollando un manejo incontrolado de todo el sistema de protección diseñado para radiología dental. 


\section{BIBLIOGRAFÍA}

1. Sources and Effects of Ionizing Radiation. United Nations Scientific Committee on the Effects of Atomic Radiation UNSCEAR 2000 Report to the General Assembly, with Scientific Annexes. Volume: Sources. New York, United Nations 2000.

2. http://www.csn.es/publicaciones/dostra.pdf

3. Vaño E, Tobarra B, Bezares M. Los datos de las exposiciones médicas en España para el informe UNSCEAR 2000. Revista de Física Médica 2000; 1: 51-60.

4. Vaño E. Las exposiciones médicas en UNSCEAR 2000 y los datos del Comité Español. Radioprotección. 2001; 30 (9): 14-9.

5. Real Decreto 2071/1995 (del. Rel. Cortes, B.O.E. 22.12.1995). Sobre criterios de calidad en radiodiagnóstico.

6. European Union. Council Directive 84/466 Euratom, laying down the basic measures for the radiation protection of persons undergoing medical examination or treatment. Official Journal of the European Communities No L 265, 5th October 1984:1-3.

7. European Union. Council Directive 96/29 Euratom, on health protection of sanitary persona and persons undergoing ionizing radiation. Official Journal of the European Communities No L 159, 29th June 1996:1-114.

8. European Union. Council Directive 97/43 Euratom, on health protection of individuals against the danger of ionizing radiation in relation to medical exposure, and repealing Directiva 84/466 Euratom. Official Journal of the European Communities No L 180, 9th July 1997:22-7.

9. Brown JE. Advances in dental imaging. Prim Dent Care 2001;8(2):59-62.

10. Smith NJD. Selection criteria for dental radiography. Br Dent J 1992; 173:120-121.
11. Horner K. Review article: radiation protection in dental radiology. Br J Radiol 1994; 67: 1041-49.

12. Rushton VE, Horner K. The impact of quality control on radiography in general dental practice. Br Dent J 1995; 7: 179 (7): 254-61.

13. Syriopoulos K, Velders X, Sanderink GC, Van Der Spelt PF, Van Ginkel FC, Tsiklak K. Mail survey of dental radiographic techniques and radiation doses in Greece. Dentomaxillofac Radiol 1998, 27 (6): 321-8.

14. Alcaraz M, Martínez-Beneyto Y, Velasco E. Control de calidad en instalaciones de radiodiagnóstico dental. Revista Europea de Odontoestomatología, 1999; 11: 265-74.

15. Rushton VE, Horner K, Worthington HV. The quality of panoramic radiographs in a sample of general dental practices. Br Dent J 1999; 12: 630-3.

16. Hirschmann PN. Justification in dental radiology. Br. Dent. J 1999; 186 (1): 9-10.

17. Havukainen R. Survey of dental radiographic equipment and radiation doses in Finland. Acta Radiológica 1988; 29: 481-5.

18. Nakfoor CA, Brooks SL. Compliance of Michigan dentists with radiographic safety recommendations. Oral Surg Oral Med Oral Pathol 1992; 73 (4): 510-3.

19. Geist JR, Katz JO. Radiation dose-reduction techniques in North American dental schools. Oral Surg Oral Med Oral Pathol Oral Radiol Endod 2002; 93 (4): 496-505.

20. Alcaraz M, Jodar-Porlan S, Martínez-Beneyto Y, Velasco E, Chiva-García F. La radiología panorámica en el radiodiagnóstico dental. Revista Europea de Odontoestomatología 2000; 12 (5): 263-70.

21. Martínez-Beneyto Y. Evolución de la radiología dental intraoral tras la instauración de la nueva legislación de control de calidad. Tesis Doctoral. Universidad de Murcia. Murcia 2003. 
22. Yakoumakis EN, Tierris CE, Stefanou, EP, Phanourakis IG, Oroukakis CC. Image quality assessment and radiation doses in intraoral radiography. Oral Surg Oral Med Oral Pathol Oral Radiol Endod., 2001; 91: 362-8.

23. Hintze H. Radiographic screening examination: frequency, equipment, and film in general dental practice in Denmark. Scand. J. Res 1993; 101 (1): 52-6.

24. Svenson B, Soderfeldt B, Grondahl HG. Attitudes of Swedish dentists of the choice of dental X-ray film and collimator for oral radiology. Dentomaxillofac Radiol 1996; 25 (3): 157-61.

25. Bohay RN, Kogon SL, Stephens RG. A survey of radiographic techniques and equipment used by a sample of general dental practitioners. Oral Surg. Oral Med. Oral Pathol 1994;78 (6): 80610.

26. Martínez-Beneyto Y, Alcaraz M, Jodar-Porlán S, Saura-Iniesta AM. Radiation protection and quality assurance in dental radiology: I. Intraoral Radiography. International Atomic Energy Agency.International Congress of Radiological Protection of Patients in Diagnostic and Interventional Radiology, Nuclear Medicine and Radiotherapy. Málaga; 2001.
27. González L, Vaño E, Fernández R. References doses in dental radiodiagnostic facilities. $\mathrm{Br} \mathrm{J}$ Radiol 2001; 74 (878): 153-6.

28. Napier ID. Reference doses for dental radiography. Br Dent J. 1999;186 (8): 392-6.

29. Cohnen M, Kemper J, Mobes O, Pawelzik J, Modder $U$. Radiation dose in dental radiology. Eur Radiol 2002; 12 (3): 634-7.

30. Williams JR, Montgomery A. Measurement of dose in panoramic dental radiology. $\mathrm{Br} \mathrm{J}$ Radiol 2000; 73 (873): 1002-6.

\section{CORRESPONDENCIA}

Miguel Alcaraz Baños

Area de Radiología y Medicina Física

Facultadd de Medicina/Odontologíá

Universidad de Murcia

30100 Campus de Espinardo

Tel.: 968363601 - Fax: 968363602

E-mail:mab@um.es 